\title{
Analysis on the Historical Role and Significance of Purple Clay Teapot and Its Inscription Decoration
}

\author{
Hongshan Chen \\ Henan University of Technology \\ Zhengzhou, Henan, China, 450000
}

\begin{abstract}
Chinese tea culture is rich in content with a long history, and as an ancient Chinese philosophical thought, the tea-culture-based "teaism" spirit is also keeping pace with the times under development and innovation. Tea set is an important content of Chinese teaism, and as a traditional tea set, the purple clay teapot has its own unique and irreplaceable characteristics: (1) The iron-rich purple clay material will generate numerous pores in the firing process, which enables the tea set possessed of both heat and freshness retaining properties and ensures that the tea or foam in purple clay teapot would not go bad after days of storage. This is the advantage that metal container, porcelain and other ceramics do not have. (2) Upon natural weathering and artificial selection, the purple clay teapot has possessed strong plasticity, which ensures that all purple clay teapots, no matter plain geometrical ones or fancy ones, are able to excellently represent the authors' thoughts and unique aesthetic appreciation. Pottery makers in Yixing had made further development based on the shape of bronze and porcelain wares, while masterpieces of masters including Gong Chun, Shi Dabin, Chen Mingyuan, Chen Hongshou, Yang Pengnian, Shao Daheng, Gu Jingzhou and so on from generation to generation had made the purply clay teapot, a practical utensil, possessed of art taste and ornamental value. (3) The participation of literati in the style design and inscription decoration of purple clay teapot makes the teapot possess dense cultural flavor and bear more profound cultural content as well as connotation of Chinese traditional culture and ethnic art culture essence. However, the purple clay teapot with inscription decoration also possesses the unique characteristics of cultural carrier and ideological connotation.
\end{abstract}

Keywords-purple clay teapot, purple clay inscription, cultural communication, individual media, accurate marketing

\section{INTRODUCTION}

Since the development of purple clay teapot, various document literature and research projects have been mostly confined to the firing of purple clay material, style inheritance and innovation of purple clay teapot and inheritance of purple clay inscription. As a teacher in design college, the author has been paying close attention to the study and practice of projects relating to Chinese tea culture, design and marketing of purple clay teapot. In this paper, analysis and research have been conducted based on the combination of the following aspects: humanism in purple clay teapot inscription design, the positive role of the purple clay teapot as a cultural carrier in communication field and the accurate marketing orientation of high-end purple clay teapot (made of refined purple clay material purely by hand).

\section{TEA DRINKING CULTURE AND TEA SET CULTURE}

\section{A. Origin and development of tea drinking culture}

As the birthplace of Chinese tea, China is the world's first country that discovered Chinese tea trees, made use of Chinese tea leaves and cultivated Chinese tea trees. It has been at least sixty thousand or seventy thousand years since the origination of tea tree and about four or five thousand years since the discovery and application of tea by human. The use of tea was first conceived in the activities of wild collection. According to legend: Shennong (patron of agriculture) tasted all kinds of herbs and one day, he ate 12 kinds of poisonous herbs but fortunately detoxicated them with tea. In ancient times, it was the tea's medical effect that made it known to the world. As the descendants of ancient "Pu people" who were the first to domesticate and cultivate tea trees, the Blang people in Yunan nowadays still talk about the story that their ancestors curbed the spread of plague by taking advantage of the detoxification effect of tea.

There has been accurate historical evidence proving that the first area to drink tea in China is Sichuan. During the period of transition from medicine to drink, the tea was constantly evolving, from unprocessed drink to dried foam drink, from steaming style to pan firing style, then from green tea to other kinds of tea. The raw tea after sunning can be fermented to produce dark tea, oolong tea and Pu'er tea, and the green tea can be processed into potpoutti tea by scenting technique. The tea in nowadays generally can be divided into six categories including green tea, white tea, yellow tea, black tea, oolong tea and dark tea along with reprocessed tea (such as jasmine tea).

Since the Three Kingdoms, Wei, Jin, Northern and Southern Dynasties, tea drinking had been divorced from medicinal use and formed a unique culture. Tea became indispensable for the emperor, nobles and aristocrats to entertain guests or even perform worship ceremony and it had been endowed with more features at a spiritual level. Following the arrival of the flourishing Tang Dynasty, Lu Yu wrote The Classic of Tea in 780, which combined the tea drinking art with the essence of Confucianist, Buddha and Taoist thoughts, elevating the tea culture to an unprecedented height and creating the Chinese spirit of teaism. 
The tea fight was quite popular among scholars in Song Dynasty, and a well-known event was the tea competition recorded by $\mathrm{Su}$ Shi. However, because the tea drinking procedures were too complicated and extravagant, the tea fight was spread to the folk but not popularized on a large scale, and the pleasure of tea fight was still enjoyed only by the nobles and scholars. In Ming and Qing Dynasties, the techniques including steaming, pan firing and baking had been basically perfect, with "handful-soaking method" for tea drinking, and tasting tea had been popular all over the country.

\section{B. Shining of tea set culture along with the diversified development of tea drinking culture}

Following the change of people's tea drinking mode, the tea set, as carrier of tea drinking, has been constantly changing. After the national unification in Sui Dynasty, the custom of tea drinking in South China was rapidly integrated with that in North China. When drinking group and cake tea, people often ground and then made the tea which was in pale yellow color after brewing. To make the tea foam easy to view, celadon ware of Yue Klin was used as the main tea set in the Central Plains, and occasionally the ceramic whiteware of Xing Klin was used, as recorded in The Classic of Tea - The Four Wares written by $\mathrm{Lu} \mathrm{Yu:} \mathrm{"The} \mathrm{porcelain} \mathrm{of} \mathrm{Xing} \mathrm{Klin} \mathrm{is} \mathrm{white,}$ which makes the tea red. The porcelain of Yue Klin is blue, which makes the tea green. It's better to use Xing porcelain than Yue porcelain."

The teaism started to spring up in Tang Dynasty and, due to the chaos caused by war in the Five Dynasties and Ten Kingdoms, revitalized until Song Dynasty when the political environment was relaxed, people's life was stable and tea fight gradually prevailed among the court and commonalty. During the tea fight which was also known as tea battle in Tang Dynasty, the exquisite semi-fermented Longfeng group tea would be used and ground into powder, if with fresh water and proper brewing, the tea would be in pure white color and the foam would be fine and undispersed for a long time. The criterion to decide the winning or losing of a tea fight not only include the bright white tea color but also include the time of foam dispersion. The one that water mark appeared first would lose. Since the tea fight in Song Dynasty required the tea color to be "white preferred", the tea set of black cloured glaze was the most suitable. Therefore, the most popular tea set at that time was from Jianzhou Kiln and Jizhou Kiln, as said in The Record of Tea written by Cai Xiang: " The white tea is better in black teacup. Wares made in Jian'an is black with fine patterns and slightly thick base, most suitable for use, far better than those made in other places which are not properly fired or in purple color. While for blue or white teacups, competitors do not use." The miraculous teacup "Yaobiantianmu" made in Jianzhou Kiln was moreover taken to Japan by the technical exchange envoy in Song Dynasty and was regarded as the "national treasure".

The blue and white porcelain had been made in Jingdezhen in the early Yuan Dynasty. As the tea drinking modes mainly included tea brewing and boiling water pouring, the tea set was dominated by teacup. In the early Ming Dynasty, Emperor Zhu
Yuanzhang who encouraged frugality issued an imperial edict to forbid the production of Longfeng group tea but only allow the contribution of picked tea bud. Thus, the Longfeng group tea stepped down from the stage of history while steamed and pan-fired loose tea was rife. Meanwhile, there had been changes in the tea drinking modes: tea brewing became the main trend at the time and was increasingly mature, and the teapot began to rise. The blue and white porcelain teapot of Jingdezhen, blue porcelain teapot of Longquan and purple clay teapot of Yixing played a leading role in the tea set of Ming Dynasty. The purple clay teapot of Yixing was deeply affected by the tea drinkers for its unsophisticated appearance as we as its breathable and heat retaining properties. In Qing Dynasty, as the governing class actively absorbed the culture of Han nationality, except the compressed tea drunk by nomads, people basically followed the tea drinking custom of Ming Dynasty. During Yongzheng Period, Yunnan Pu'er tea arouse, which has enriched the variety of Chinese tea.

Along with the development and progress of technology as well as the improvement of people's living standard in nowadays, the way of tea drinking has gradually shown the tendency of diversification. In addition to the inheritance of traditional modes, foreign drinks like broken black tea, tea bag, herb tea, coffee and cocoa and so on have entered people's life. While the tea set has achieved significant progress, developing from ceramic products to high-tech electronic tea stoves, also including teacups made of metal, glass, wood, paper and plastics; actually, all wares, from exquisite design works to disposable containers, can be used as tea set.

It is true that the disposable tea set is quite convenient and the instant and tea-bag drinks are more consistently in line with the fast-paced urban life, which, however, are lack of connotation and fun compared with the tea leaves and tea sets bearing the Chinese culture. The traditional tea set, taking purple clay teapot as an example, will allow people to enjoy the model beauty of purple clay teapot, touch the texture beauty of teapot body and taste the conception beauty of inscription during tea making as well as to feel the beauty of improved tea flavor brought by purple clay teapot during tea tasting.

The development of tea drinking culture has provided the foundation for the emergence and development of tea set. As the new way of tea drinking is always leading to the occurrence of corresponding tea set, the development of tea set is inseparable from that of tea drinking culture. For instance, people can enjoy the loose green tea gently floating and unfolding in a transparent glass, as if watching tea dance. The delicate Kung Fu black tea can be made by glass retainer cups. At present, the six categories of tea in China all can be made by one kind of tea set, namely the purple clay teapot. The iron-rich purple clay material will generate numerous pores in the firing process, which enables the tea set possessed of both heat and freshness retaining properties and ensures that the tea or foam in purple clay teapot would not go bad after days of storage. This is the advantage that metal container, porcelain and other ceramics do not have. And for this reason, the tea 
making by purple clay teapot can not only increase the tea foam temperature to release the tea aroma but also preserve the activity of tea leaves to ensure no production of hard-boiled tea foam.

\section{DEVELOPMENT AND ROLE OF PURPLE CLAY TEAPOT}

The purple clay teapot of Yixing characterized by the "ability to preserve the color, aroma and flavor of tea" shines more brilliantly in the company of teaism sprit, hence firmly occupying a place among China's traditional tea sets. It's inevitable to discuss the purple clay teapot when speaking of Chinese tea sets.

The historical celebrities and the development trace of purple clay teapots.

Yixing purple clay teapot as a tea set can be dating back to Zhengde period of the Ming Dynasty, and was first created by Gongchun and the monks of Jinsha Temple ( refer to the Yangxian Teapot Series of Zhou Gaoqi in the Ming Dynasty). At that time, people praised Gongchun teapot that "The color of purple clay teapot $\mathrm{s}$ is dark with red, just like the color of iron, and the teapots look plump and decorous."

After Gongchun, the artisans of purple clay teapot became more and more in Yixing. Until the period of Wanli, the design of purple clay teapot achieved a new development again. Xu Cishu in the Ming dynasty said in Tea Note that "The teapots made by Gong Chun in the past, and the teapots made by Shibin recently, are admired and cherished by people at the same times." Shi Dabin can be considered as a master linking between the preceding and the following in mud preparation, shapes techniques, style design, inscription and other aspects. Today, the works of Shi Dabin that are handed down, including the three-leg round-lid teapot, the teapot engraved with belt-liked dragon in imitation of Gongchun teapot, the mitre-liked teapot, the hexagonal teapot, and the Tianxing pavilion-liked teapot, all are fine works. When Shi Dabin early made teapot, he always asked people to write words and then engraved the words. Later, he practiced writing hard without stop, and finally he can write words by using bamboo sword instead of writing brush. Li Jingkang and Zhanghong in the Republic of China(1912-1949)wrote in Yangxian Teapots in Photographs and Measured Drawings that " Early Shi Dabin engraved inscription on the teapot by bamboo sword based on the script of writer and later he was able to engrave inscription directly by sword without others' descript. His handwriting is skilled and elegant in Hang Ting's and Yue Yi's copies, and no one is unable to imitate, used by connoisseurs for identification." Shi Dabin makes teapot delicately on his own, and even cultivated disciples such as $\mathrm{Xu}$ Youquan, Li Zhongfang, etc.

Wars went on during the late Ming Dynasty and the early Qing Dynasty.

After the society becomes stable in the mid KangXi's period, the design and making of purple clay teapot steped into a new era, and Chen Mingyuan is a representative of this era. Chen Mingyuan, styled Mingyuan, was also known by his literary name Hefeng, Shixia Hermit or Teapot Hermit, a famous artisan of purple clay teapot in Yixing during the period of KangXi's reign of the Qing Dynasty. Cheng Mingyuan does not follow the stereotypes, and has courage to be the first, thus he rebuild the abstract production of purple clay teapot, and brings the nature into the teapot. His teapots was full of great fun of life, such as the pine-bamboo-plum-liked teapot, the muskmelon-liked teapot, the pine-section-liked teapot, the plum-branch-liked teapot etc. The desk displays in study designed by him are elegant and vivid, lifelike and amazing, which are in models of water chestnut, haricot bean, peanut, maize, chufa, lotus root slices, walnut, and others. Chen Mingyuan made the natural teapot shape in the Ming dynasty full of art, making it more alive and fresh, thus opened a precedent of plain geometrical teapots for aftertime. He also introduced the decorative art of traditional painting and calligraphy and the inscription way into the craftsmanship of purple clay teapot, making the original plain teapots add many meaningfully decorative taste, and also making the purple clay teapot have strong bookish. Besides, the calligraphy of inscriptions is beautiful and graceful, rich in writing significance of Jin and Tang dynasties, integrating the pot art, tea, and literati's temperament and interest into one, to greatly improve the artistic value and cultural value of purple clay teapots. Thus purple clay teapots become real works and enter into art galleries.

Chen Hongshou, styled Zigong, was also known by his literary name of Mansheng, Mangong, Gongshou, Manshou, Laoman, with alias of Zhongyu Hermit, Yanggu Official, or Xu Xiyu Hermit, and A Man Tuo Shi was the usual seal of his purple clay teapot. Chen Hongshou was born on the thirty-third year of Qianlong period, a flourishing age of calligraphy and painting creation, with primary school and epigraphy popular. His calligraphy, painting and inscription all obtained outstanding achievements. As an inheritor of QianJia School, Chen Hongshou "likes imitation and inscription very much in life" (extracted from Artists Biography). Chen Mansheng was addicted to copying inscription and good at seal engraving. He had excellent skills, and thus he becomes one of eight engraving masters of Zhijiang Seal School. Chen Mansheng's seal engraving pursued the way of flat and simple Han Seal, not trying for novelty and innovation. He respected nature "all the poets, calligraphies and paintings should not be perfect, but show natura; interest. Because of this kind of artistic value of natural interest, Chen Mansheng, who liked purple clay teapot at his early years, when he was the county magistrate in Liyang County, met Yang Pengnian who was a craftsman of purple clay teapot at that time. From the Emperor Qianlong period in the Qing Dynasty, the pattern pots that were molded in segment, became popular, for such pattern pots could save time and be produced in a large quantity. But Yang Pengnian still persisted in making pots by hands, for which the deceased Gu Jingzhou, an industrial arts master, give him an appraisal of that "Pengnian's skills of pot arts are ordinary and not outstanding." At that time, Chen Mansheng has made remarkable achievements in the field of painting and calligraphy carving, and he chose Yang Pengnian with ordinary skills as his partner just for Pengnian's honesty and nature in pots. This similar artistic value created the renowned "Mansheng Pots", no one comparable afterward. Mansheng pot has unsophisticated and natural style, with its inscription 
on the pot body coming to the point of pots, tea, water, and feeling, so it was appreciated and admired by the appreciators of contemporary and future generations.

In the period of Daoguang and Xianfeng in the Qing Dynasty, Shao Daheng, a famous maker of purple clay teapot followed the sound, plain and generous way, and he has unique craftsmanship in his works such as Duozhi, Yuhualong, LongtouYikunzhu and other teapots. It was said in the Theory of Teapot . Presenting to Shao Daheng by Gao Xi in the Qing Dynasty that "Shao Daheng, good at teapot making, with its elegant style but unfixable, was apt to antique imitation and emulating the ancients, often surpassing the former. His pick-up pot has even and sound shoulders, neck and abdomen, suiting both refined and popular tastes, not ridiculed by any one but appreciated by more and more people. Its wonderful and natural for pouring tea, especially unsophisticated and elegant. The lid is just and tight, and even if declined, it couldn't drop. The mouth is thick and narrow inside to prevent the imperfection. The air hole is small outside and subuliform inside, like a loudspeaker, so there is no disadvantage of obstruction.

From the late Qing Dynasty to the Republic of China, domestic trouble and foreign invasion were continuous. Although a group of purple clay teapot celebrities emerged in Yixing, such as Huang Yulin, Cheng Shouzhen, Yu Guoliang et al, whose workmanships were masterly, and they have their representative works, they have no style of great masters, compared with the elder masters .

After the founding of new China, the purple clay industry was about to be revitalized. A group of skilled and lettered young purple clay artists full of energy stood out and created the new peak of purple clay art. The outstanding ones include Wu Yungen, Zhu Kexin and Gu Jingzhou. Born in 1915, Gu Jingzhou studied in Shushan Dongpo Academy, he was exceptionally smart and was often praised by the mentor. At the age of 18, he followed in his ancestor's footsteps at hometown, learning Shao's blank making from his grandmother and meanwhile inheriting the pot-making techniques from the home guest teacher. With a firm knowledge of literature, he had made remarkable works and became famous overnight, joining the ranks of the renowned pot-art masters. When he was 20 , he was hired by Shanghai Lang's Art Gallery to make pottery by imitating ancient works. With superb skills, he imitated the Chen Mingyuan's (of Qing Dynasty) Longfeng Bazui Pot and Zhusun Jar which had been unexpectedly regarded as the legacy of Cheng Mingyuan and collected by the Palace Museum and Nanjing Museum. Until decades later, when $\mathrm{Gu}$ Jingzhou was conducting verification over the purple clay collections in the Palace Museum and Nanjing Museum, he discovered that they were his own works. At the middle age, $\mathrm{Gu}$ Jingzhou strove to break new ground, integrating the modern aesthetic elements into his works. The pots he had made during this period, including Hanyun, Xuehua, Zhegu, Tibi, Shangxinqiao, Fengzuihu, Sengmao, Fanggu, Shipiao, Niugai Lianzi, etc., all had unsophisticated and elegant overall design, robust and rigorous body and smooth and harmony line, manifesting strong Oriental art characteristics, with infinite profound meaning and varied presences, free from vulgarity, which deserved to get the comment "integrating the essence of clay arts and refreshing the delicate and complicated style". Of which, Niugai Lianzi and Zhegu won the prize of National Gold Award, and lots of excellent works were collected by collectors and museums at home and aboard. Gu Jingzhou's superb skills and profound cultural accomplishment enable him to inherit the classics and innovate the pattern. He dedicated his lifelong effort in purple clay pottery, wrote the Introduction to the History of Purple Clay Pottery and complied Appreciation of Purple Clay Works of Yixiang in his later years. Gu Jingzhou took numerous apprentices who had accomplished great achievements, such as Xu Hantang, Gao Haigeng, Li Changhong, Zhou Guizhen, Pan Chiping, etc. In the history of Chinese purple clay development, Gu Jingzhou is undoubtedly of the great importance of a milestone, as the famous artist Mr. Yaming once remarked: "It has been about five hundred years since the occurrence of purple clay wares in Zhengde Period of Ming Dynasty, but the number of maters in this filed is only more than ten. Mr. Gu Jingzhou fully deserves the title of modern master and his works have perfectly expressed the philosophical spirit, literature flavor and painting charm of China."

Because arenaceous clay possesses a strong plasticity through natural weathering and manual screening, the fired purple clay teapot, regardless of plain geometrical teapots or fancy teapots, may well reflects the author's ideas and unique aesthetics. Potters in Yixing carried out further development according to shapes of bronze, porcelain and other wares. Moreover, the aborative works by generations of masters, such as Gong Chun, Shi Dabin, Chen Mingyuan, Chen Hongshou, Yang Pengnian, Shao Daheng, Gu Jingzhou etc. further made this practical ware purple clay teapot possess artistic taste and ornamental value.

\section{THE DEVELOPMENT TRACE AND THE ROLE OF PURPLE CLAY TEAPOT FOR ITS INSCRIPTION}

Making inscription at the bottom of shaped artifacts has always been a measure to indicate the source of artifacts but not undermine the overall aesthetic sense of artifacts. Ceramic craftsmen should ever think of the decoration of calligraphy and painting on the surface of artifacts. However, first, they are lack in cultural knowledge foundation, and second, craftsmen and scholars are in different two stages and both can not exchange their ideas, so as to not make perfect combination of artifacts and decorative paintings. Purple clay teapot is especially thus, because only teapot craftsmen know the pot contraction scale when teapot is fired into the kiln for one time, but the calligraphists and artists without further accomplishments of inscriptions are unable to engrave inscription or painting on the teapot body with bamboo sword, and even if they dare to engrave inscription, they are unable to foreknow whether the work may be deformed severely and teapot craftsmen can not guarantee the artistic mood of paintings is reflected on the pot body. This case is not changed until Chen Mingyuan comes forth. As a pioneer of the era, the great master combines the genre, layout, typeface, font, inscription, mood and many other elements of pot body decoration with the pot type, taking it easily generally, to make a perfect combination of practicality and cultural richness of teapot, and to improve the artistic quality of teapot, so that all 
workshops are proud of getting a teapot portrayed by Chen Mingyuan. It is recorded in "YangXian Famous Pottery List" by $\mathrm{Wu}$ Qian, in Qianlong's reign of the Qing Dynasty that "Mingyuan has a skill, born in an aristocratic family of purple clay teapot, with its outstanding work among others published. For hundreds of years, few are comparable with his works, so he becomes famous. Wherever he arrived, literators and scholars strive for inviting him. He often went to Zhang's Sheyuan Garden in Haiyan, Wang Keting's family in Tongxiang, Cheng's, Cao's and Ma's families in Haining, where his work most can be found, and he has especial friendships with Yun Wanyan in Yangzhong. I had got Mingyuan's one work "Chicken- over-sky Teapot", luxurious and elegant, made of fine sand, black with red, with "Anthology of Yuzi Mountains" engraved from the script of Mr. Cao Lianrang, comparable with the three generations of ancient artifacts. Contend it with Dabin's teapot to fear to be listed second such as Zhu state and the Ju state."

With a considerable talent for making Yixing Purple Clay Teapots, Chen Mingyuan was deeply affected in the communication with literary men and scholars, and thus his accomplishments in culture and art was improved to an extremely high level as well. When visiting Wang Xishan, Zhang Yanchang saw a Yixing Purple Clay Teapot made by Chen Mingyuan, with an inscription at the bottom which reads: 'The pleasure of Confucious and Yanhui lies in nothing but drink fragrant tea.' After reading it, Zhang Yanchang was impressed a lot and said: 'What Mingyuan inscribes here is awesome, and isn't he a man with high taste hidden in potters? Chen Mingyuan's skill in making teapots and his inscription shines more brilliantly with each other's accompany. The Melon Teapot kept in Nanjing Museum now is engraved with verses 'The shape of the Teapot is imitating that of Dongling Melon; the tea from it smells extremely fresh and fragrant.' The teapot boasts a natural shape and masterful calligraphy. In the verses, Chen quotes a historical allusion that in the early Western Han Dynasty, Dongling Marquis of the previous Qin Dynasty degraded himself to be a poor farmer and made a living by planting melons which were called 'Dongling Melons'. Through the allusion, Chen voices his indifference to fame and wealth. The Four-leg Teapot made by Chen Mingyuan is kept in Shanghai Museum. On its body there is an inscription 'When you feel full that is enough no matter it's for sipping tea or reading', which indicates his humility. His Lotus Teapot is inscribed with the words 'It will bring you nobility and drive downers and heat away from you; with such a quality, noble men will be friends with you for ever.' Therefore, Chen Mingyuan's teapots have been transformed from utilitarian handicrafts into the carriers of cultural and art. Art and the delight brought by teapots supplement each other. Yixing Purple Clay Teapots made by Chen Mingyuan not only present his artistic value, but also are used to express his thoughts and ideas.

Not every potter can have literary accomplishments, especially in feudal society. At that time, craftsmen and literary men were not categorized into the same class, and craftsmen were usually illiterate while literary men were too proud to design the Purple Clay Teapots by themselves though they love the teapots so much, because their final aim is to be officials. As a result, after the era of Chen Mingyuan, Purple Clay Teapots were made elaborately and fancily for a long time. What became popular were the crafts that focusing on gorgeous appearance instead of the low-heat glaze imitating Jun Kiln with which Purple Clay Teapots are endowed. Literary men and the group enjoy sipping tea suffered a lot from the abnormal aesthetic of Purple Clay Teapots. Under such a circumstance, Chen Hongshou emerged.

Chen Hongshou advocates 'natural delight' and exploiting the natural beauty of Purple Clay Teapots. He ushers the inscription and decoration on the Purple Clay Teapots into a new path.

The calligraphy and seal cutting by Chen Hongshou follows the tradition and also seeks innovation so as to obtain natural delight. He is motivated by Moya Stone Tablets and achieves great accomplishment on the small body of teapots. Taking earthen bricks as paper and engraving on them, he presentes the natural delight of purple clay completely.

Chen Hongshou introduced a new method to later literary men and craftsmen of Purple Clay that literary men can cooperate with congenial craftsmen. In his cooperation with Yang Pengnian, Chen Hongshou was spared from making teapots by himself. Although Yang's skill was not so perfect, he could make teapots exactly as designed by Chen Hongshou and gave them special charm. With more and more cooperation and adjustment, Yang even could feel the natural delight included in the design of Chen Hongshou and embodied it in his works. Take the calligraphy and painting masterpiece 'Chrysanthemum and Teapot' as an example. It has two inscriptions in running script. The right one tells us that Yang is good at making teapots, and he loves teapots and takes making teapots as a hobby, but can't make them well. The teapot in his painting is perfect in his heart. The left one reads 'Tea is ready; chrysanthemum is blooming; will you come here to enjoy the autumn scenery?' By Mansheng (the literary name of Chen Hong shou). From them, we can know that Yang earns his recognition.

Though in different artistic styles, Chen Mingyuan and Chen Hongshou, for inscription and decoration of Purple Clay Teapots, have pioneered two roads that lead to the same destination. One is to improve the cultural accomplishments on the part of craftsmen, designing teapots and adding inscription and decoration when making them. The other is to cooperate with each other, literary men being engaged in designing and inscribing while craftsmen making teapots as designed. All craftsmen of later generations follow the two methods to produce teapots with inscription and decoration.

When the skills are mature, the Purple Clay Teapot has a new function as a cultural carrier combing literature, calligraphy, painting and seal cutting together and as a platform to transmit the individual artistic thoughts. The rising of purple clay inscription and decoration created a new trend that is the teapot becomes more valuable due to the inscription on it, and the inscription is transmitted along with the teapot.

\section{A. The Problems in the Development of Purple Clay Inscription and the Solutions}

Good inscription and decoration can always add value of 
the Purple Clay Teapot. On the Purple Clay Teapot of Yangxian(what Yixing was called in Qin and Han Dynasties), a book written in 1937, writes 'a common teapot is only worth 240 wens (wen, an ancient monetary unit), but if it is added with inscription and decoration, the value will triple.' Despite the fact that art should not to be measured by money, a work of art can be judged to be good or not through its price. Nowadays, some craftsmen are too eager for great success and instant benefits. They make teapots quickly without full devotion into them, go to famous calligraphers and painters for inscription and painting, and then sell them out at a high price. What the inscription and painting describe have nothing to do with the shape of Purple Clay Teapots, let alone close association with tea and feeling. Such a shortsighted behavior is not beneficial to developing Purple Clay crafts or improving the aesthetic level of craftsmen. To change the phenomenon fundamentally, craftsmen should make efforts as follows: Firstly, to improve personal artistic accomplishments and understand the rich cultural elements in the inscription. Take Mansheng Teapot as an example, craftsmen should know about the inscriber's psychology and inscription. Secondly, to position the market accurately, categorize the customers elaborately and conduct precise marketing.

The verses in the inscription of Mansheng Teapot are both elegant and interesting, matching perfectly with its shape. Semicircular Moon Teapot is named due to its shape---a semicircular moon. On its body is inscribed 'The moon waxes only to wane, which will be posted at the right hand of my seat and regarded as my motto.' The inscriber warns himself not to be arrogant and that humility is a virtue.

Square Teapot is inscribed with 'It is clear inside and square outside, and so we should.' Here, it not only depicts the shape of the teapot, but expresses the inscriber's moral standard: upright and foursquare, comprehensive when thinking about something.

Joyful Teapot is inscribed with 'to brew Yangxian tea with Hejiang water, and such joy is people such as Su Dongpo( a famous literary man in Song Dynasty) are pursuing for.' Using the allusion of $\mathrm{Su}$ Dongpo, the inscriber explains the real joy lies in nothing but everyone's joy. In another word, it is more joyful to share the joy than to keep it to oneself.

The basic demand for inscription is that it should accord with the shape and artistic conception of the teapot. No matter for teapot imitating ancient shapes or innovative ones, the inscription and decoration have to manifest their artistic conceptions. Only in this way, can a teapot show outstanding artistic qualities and unique cultural scents, can a teapot become an artwork in stead of a simple handiwork.

\section{B. The precision marketing of high-end purple clay teapot with inscription}

Compared with traditional plain geometrical teapots or fancy teapots, purple clay teapot with inscription is more aimed, and has smaller consumer group. A purple clay teapot represents the author's aesthetic tendency, with its shape and inscription both reflect the author's aesthetic perception of this teapot. Whether the teapot is customized by appreciators, or is caused by maker's interest, its consumer group must not be the mass. It is said that the benevolent delight in the mountain, the wise delight in the water, so the reason of buyers to buy is that it can lead to psychological resonance. A high-end manual purple clay teapot finely made of valuable mud, with appropriate inscriptions, must be followed by the buyer with unique discernment, and the value of the author and the works is also reflected.

\section{The social value of purple clay teapot with inscription as the media}

As one of traditional Chinese tea sets, although its arisen time is not long, it is the most suitable tea set for China's tea, and its physical characteristics and cultural characteristics fit perfectly with Chinese tea culture. The inscription of purple clay teapot, as the best line appearing in purple sand arts, makes the purple crafts harmonized and unified with Chinese traditional culture, and shows the essence of "ware" in Chinese tea culture.

Since Chen Mansheng, literati have been increasingly keen on the shape design and inscription decoration of purple clay teapot. They design the style of purple clay teapot with their own philosophy, and enrich the inscription decoration of purple clay teapot with skills, such as painting and calligraphy, etc. They make purple clay teapot as a platform for carrying and spreading personal ideas, and show the inner world to the specific crowd by the way of inscription. This transmission way is similar to a point-to-point transmission way, and becomes commonplace in today's network platform. BBS, blog, podcast, micro blog, SMS, and other communication tools, making everyone may become the object of strangers' attention. Personal media, the media, and other transmission way which are different from mass communication, has become new things. In fact, a few hundred years ago, the inscription decoration of purple clay teapot has preliminarily had the function to spread towards specific objects in small group. The inscription decoration of purple clay teapot releases the content that mainly covers motivational inscription, gloss, motto, and the discussion of outlook on life and values. For the publishers are famous literati, at that time, these contents played an active role in the society to stimulate thinking, enrich cultural life, broaden horizons, and guide the ideological trend.

\section{CONCLUSION}

Literati participate in the design of shape and inscription of Purple clay teapot, which makes purple clay teapot possess more deep culture, and carry more rich cultural content, Chinese traditional culture and the essence of national art. Therefore, purple clay teapot with inscription decoration also possesses the cultural carrier, ideological implication and other characteristics which are different from other tea sets. It is these characteristics that make inscription of purple clay teapot appear in the history as a kind of personal media, and constantly inherited. Today's purple artists have a responsibility to do painstaking research on this unique cultural form and carry forward, making the inscription become the best line and the essence reflection of purple clay teapot, rather than artily impose some irrelevant words on a piece work and thus make the work lose its soul. 


\section{REFERENCES}

[1] Xu Xiutang. China's Purple Sand. Shanghai Ancient Books Publishing House. 2007

[2] Han Qilou. The Book of Purple clay teapot . Fujian Arts Publishing House, 2004

[3] Shi Juntang. Purple Research. Shanghai Ancient Books Publishing House. 2007

[4] Xu Xiutang. Xu Xiutang telling of Purple clay teapot . Shanghai Dictionaries Publishing House. 2007

[5] Liu Zongyue. The craft culture. Guangxi Normal University Press. 2006

[6] Gu Jingzhou. Yixing Teapot appreciation. Joint Publishing. 1992

[7] Gao Yingzi. Zisha Pottery Book. Zhejiang Photographic Art Publishing House. 2000

[8] Qian Jianhua. The Shape and Appreciation of Purple clay teapot . Jiangsu People's Publishing House. 1992

[9] Cha Junfeng. Tea Culture and Tea sets. Sichuan Science and Technology Press. 2004.

[10] Pan Jialai. Chinese Traditional Purple clay teapot . People's Fine Arts Publishing House. 2003

[11] Sun Weizu. Chen Hongshou Carving. Shanghai Bookstore Publishing House. 2007

[12] Zhu Hongjun. On Engraving Style and Impact of Mansheng Teapot. Jiangsu Ceramics. 2006 